\title{
Health Professionals' Perceptions and Reactions to ICT-Related Patient Safety Incidents
}

\author{
Jouni KOUVO ${ }^{\mathrm{a}, 1}$, Samuli KOPONEN ${ }^{\mathrm{a}}$, Hanna KUUSISTO ${ }^{\mathrm{a}, \mathrm{b}}$ and Kaija SARANTO ${ }^{\mathrm{a}}$ \\ ${ }^{a}$ University of Eastern Finland \\ ${ }^{\mathrm{b}}$ Tampere University Hospital, Department of Neurology
}

\begin{abstract}
Patient incident reporting is an important way to promote safer health care. The barriers for reporting can be organizational (leadership, culture, lack of feedback, etc.) or individual (time pressure, perceived competence, attitude, etc.). In this study, we examined what kinds of ICT-related incidents health professionals observe in Finland, how they react to them and the reasons for non-reporting. Our data was collected using a nationwide survey during the Spring of 2020. The theory of planned behaviour by Ajzen served as our framework for explaining nonreporting behaviour. While we found that attitudes, subjective norms and perceived behavioural control all explain non-reporting, our factor model based on our confirmatory factor analysis did not directly match Ajzen's theory.
\end{abstract}

Keywords. Health care informatics, patient incident reporting, the theory of planned behaviour.

\section{Introduction}

The importance of patient safety and the need for safer health care practices became apparent after a leading-edge report by the Institute of Medicine in 2000 [1]. Patient safety incidents are a major concern also in Finland, with varying consequences ranging from damage to an institution's reputation to loss of lives. Finland introduced a national incident reporting system in 2007 and the current trend is a yearly increase in incident reporting by health professionals [2], indicating an increasing awareness of patient safety culture. However, there is still room for improvement, especially since new patient safety risks are emerging, many of which are related to information systems and communication [2].

There are organizational barriers to reporting patient safety incidents. Reporting very rarely leads to recommendations, let alone their implementation [2]. Lack of transparency and feedback decreases willingness to report incidents $[3,4,5]$, as well as insufficient managerial support [3,4] and uncertainty about what types of events and what level of severity should be reported [3, 4, 5]. A punitive culture and fear of consequences can also be barriers to incident reporting $[4,5]$.

\footnotetext{
${ }^{1}$ Corresponding Author, Jouni Kouvo, University of Eastern Finland, P.O.BOX 1627, 70211 Kuopio, Finland. E-mail: jouni.kouvo@gmail.com
} 
One of the most popular socio-cognitive theories to predict and explain behaviour is the theory of planned behaviour [6,7]. According to the model, behavioural intention predicts actual behaviour and three factors affect behavioural intention: attitude (beliefs about the consequences and experiences of behaviour), subjective norms (beliefs about the expectations and behaviours of others) and perceived behavioural control (beliefs about resources and opportunities) [8]. While there is evidence that the model predicts patient incident reporting intentions quite well $[9,10]$, there is no clear understanding as to which factors have the greatest impact $[11,12]$. The model has also been expanded with other psychological concepts, such as altruism [12], psychological safety [10] and self-efficacy [10].

Our research questions are: 1) What kind of ICT-related patient safety incidents do health professionals experience in their work? 2) What kinds of actions do they take when they are noticed? 3) What are the reasons for not reporting incidents? 4) Do attitude, subjective norms and perceived behavioural control explain behaviour, as predicted by the theory of planned behaviour?

\section{Methods}

In Spring 2020, the Finnish Institute for Health and Welfare conducted a nationwide survey (STePS 3.0) on information system services in health care. An anonymous web questionnaire was sent to 58276 health care professionals, of whom 10094 opened the link and 3912 completed the survey. The 3610 replies were sufficient for the analysis, representing $35.8 \%$ of those who opened the link. The survey was designed to assess how users experience information systems' functionality, usability, and support for daily practice, as well as to describe the current status and needs for improvements of the electronic health care system [13].

As some of the questions were directly related to patient safety incidents and their reporting, the following variables were included in this study:

- "If during the last 12 months you have noticed patient safety incidents caused by use of information systems, what kinds of errors occurred?" (See options in Figure $1)$.

- "What did you do when you noticed incidents?" (Figure 2).

- "If you didn't report incidents, what caused you to make that decision?" (Figure 3).

Data analysis was carried out with SPSS (version 25.0) and Amos, and it included descriptive and inferential statistics. For inferential statistics, we used principal component analysis and confirmatory factor analysis.

\section{Results}

Altogether $92.5 \%$ of the participants were women, and most of them worked as a nurse or similar $(78.1 \%)$ in a public-owned organization run by a municipality $(85.2 \%)$. Age was more evenly spread, with a majority of the respondents being born in the $1960 \mathrm{~s}$ $(30.6 \%)$ or $1970 \mathrm{~s}(28.2 \%)$. 
Almost all the participants had experienced at least one ICT-related incident during the last 12 months $(94.6 \%)$. A majority of incidents were caused by human errors, not system malfunctions. The most typical adverse findings were related to medication lists or patient registrations (Figure 1).

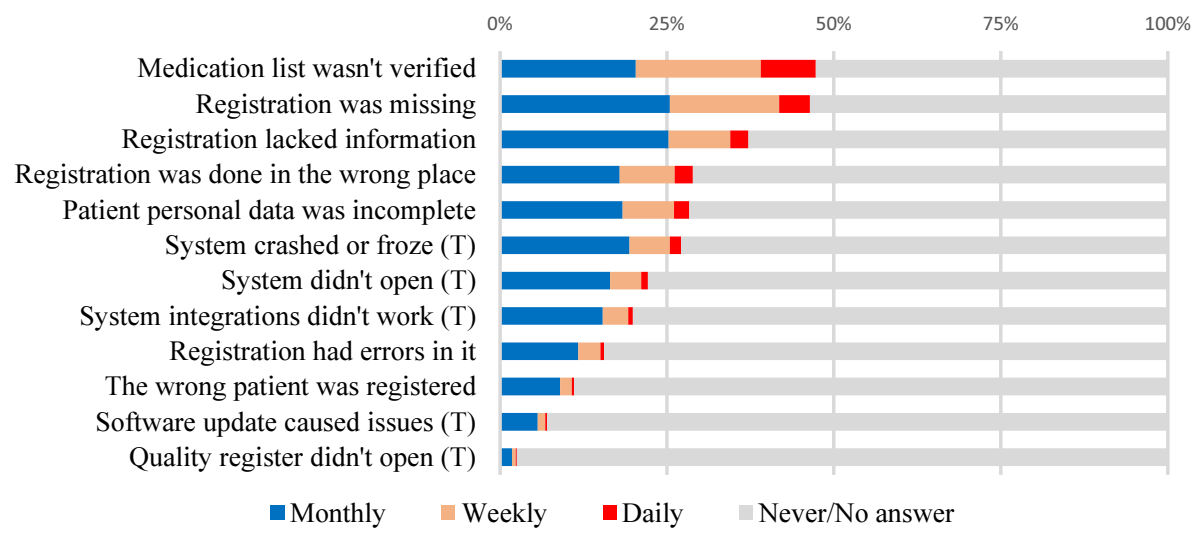

Figure 1. ICT-related patient safety incidents observed by health professionals during the last 12 months $(\%) .(T)=$ Technological issue, not human mistake. $(\mathrm{N}=3610)$.

When health professionals witnessed an incident, the most common action they took was to discuss it with their colleague or manager (Figure 2). In almost half of the cases $(45.7 \%)$ they created a patient safety incident report. Principal component analysis showed that there were three different patterns of reactions (in order of popularity): 1) Discussing (with colleague, manager or patient), 2) Reporting (in the patient incident reporting system) and 3) Contacting (help desk or super user). A KMO measure of .612 and Bartlett's test $\mathrm{p}<.001$ suggest that the model is appropriate.

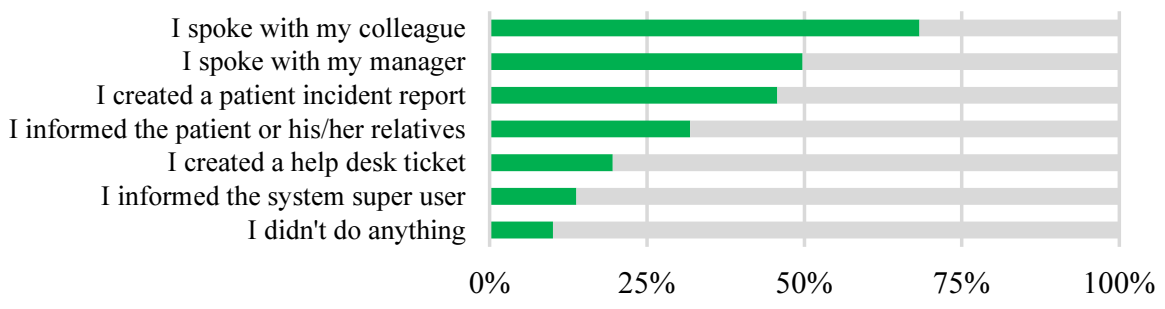

Figure 2. Actions taken with regards to ICT-related patient safety incidents $(\%)$. $(\mathrm{N}=3158)$.

The last questions covered health professionals' explanations for not reporting (Figure 3). About half of the respondents said that they did not have time to report or they did not report because no actual harm was done to the patient. Approximately $10 \%$ of the people felt that their organization did not expect them to report patient safety incidents or that they did not even have access to a reporting tool. 


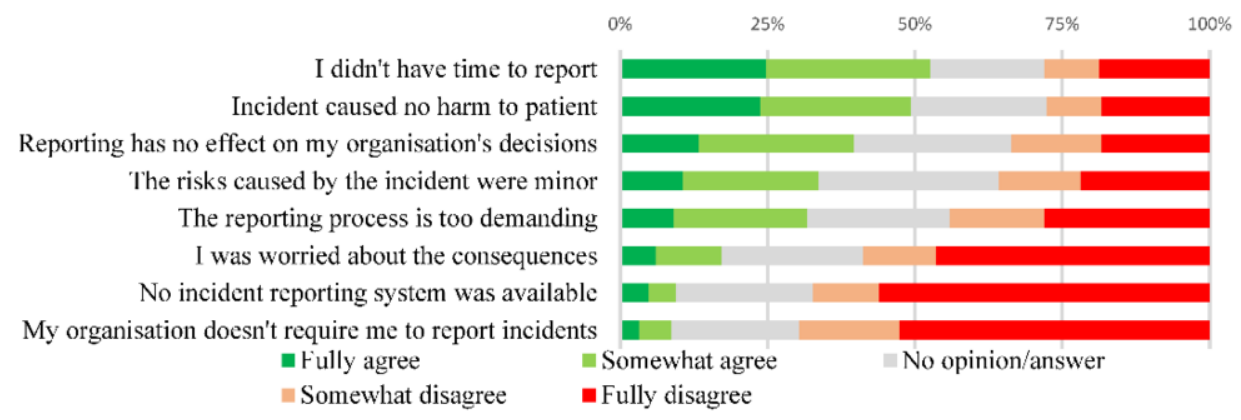

Figure 3. The reasons for not reporting ICT-related patient safety incidents (\%). $(\mathrm{N}=3022)$.

Confirmatory factor analysis was conducted to see how the indicators loaded on predicted factors and how the factors were correlated. The following three dimensions were identified:

- Subjective norm (perceived expectations of the organization)

- Attitude (beliefs about the need to report incidents)

- Costs vs. Benefits (the amount of time and effort needed for reporting vs. the expected benefits)

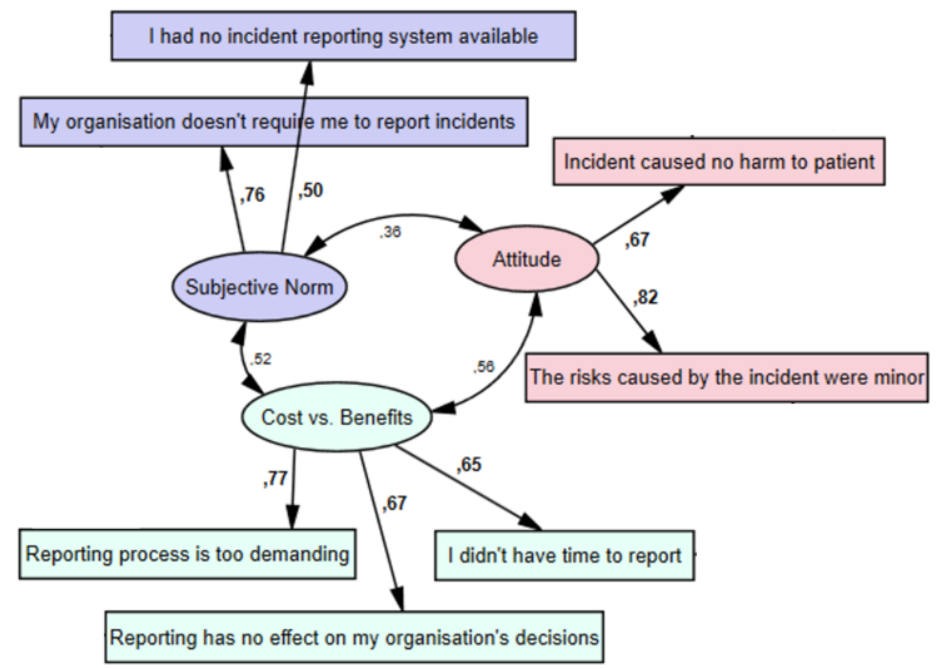

Figure 4. The factors for not reporting ICT-related patient safety incidents (\%). ( $\mathrm{N}=3022$. RMSEA .067, NFI .967, IFI .969, CFI .969).

\section{Discussion and Conclusion}

Practically all participants $(\mathrm{N}=3610)$ had observed ICT patient safety incidents during the last 12 months. Incidents were mainly caused by human mistakes, so it is understandable that the most typical reaction was to discuss the matter with a colleague or manager. However, in almost half of the cases respondents created an incident report, which confirms the findings that reporting in Finland is at a quite good level [2]. 
For non-reporting, we could not find all three factors predicted by Ajzen's theory. Instead of perceived behavioural control, we found a factor which could be called costs vs. benefits. Literature has shown that a lack of feedback and recommendations decrease reporting willingness [2-5]. It is interesting that in our study, respondents seemed to have considered available resources (time, competence) and expected outcomes together.

We did find a component representing a subjective norm, i.e. insufficient managerial support, which is known to negatively affect reporting $[3,4]$. Our data also confirmed that people sometimes skip reporting because they consider events as not severe enough [3-5]. That may indicate an attitude issue or lack of proper instructions.

The three components of the theory of planned behaviour are known to covary and their exact impact is still unclear [7,11,12], so further research is needed. It would also be interesting to compare how the model works for different groups of people in health care: public vs. private sector staff, nurses vs. physicians, etc.

Our questionnaire was crafted and reviewed by scholars from various Finnish research organizations, and our sample size was quite large (3610), so we can assume adequate reliability of this study. However, external validity of our results is limited: they cannot be directly generalized outside the (mainly public) nursing community in Finland. Lastly, we did not use pre-existing scales from the literature to measure some of our key concepts, such as attitudes or subjective norms, which might compromise our study's internal validity.

\section{References}

[1] Kohn LT, Corrigan JM, Donaldson MS. To Err is Human: Building a safer health system. Institute of Medicine Report. Washington, DC: National Academy Press. 2000.

[2] Liukka M, Hupli M, Turunen H. Problems with incident reporting: Reports lead rarely to recommendations. Journal of Clinical Nursing. 2019;28(9-10):1607-1613.

[3] Pfeiffer Y, Briner M, Wehner T, Manser T. Motivational antecedents of incident reporting: evidence from a survey of nurses and physicians. Swiss Medical Weekly. 2013;143; w13881.

[4] Winsvold Prang I, Jelsness-Jørgensen L. Should I report? A qualitative study of barriers to incident reporting among nurses working in nursing homes. Geriatric Nursing. 2014;35(6):441-447.

[5] Rashed A, Hamdan M. Physicians' and Nurses' Perceptions of and Attitudes Toward Incident Reporting in Palestinian Hospitals. Journal of Patient Safety. 2019;15(3):212-217.

[6] Godin G, Belanger-Gravel A, Eccles M, Grimshaw J. Healthcare professionals' intentions and behaviours: A systematic review of studies based on social cognitive theories. Implementation Science. 2008; 3:36.

[7] Ajzen I. The theory of planned behaviour: reactions and reflections. Psychology \& Health. 2011;26(9):1113-1127.

[8] Ajzen I. The theory of planned behavior. Organizational Behavior and Human Decision Processes. 1991;50(2):179-211.

[9] Ben Natan M, Sharon I, Mahajna M, Mahajna S. Factors affecting nursing students' intention to report medication errors: An application of the theory of planned behavior. Nurse Education Today. 2017; 58:38-42.

[10] Lee Y, Yang C, Chen T. Barriers to incident-reporting behavior among nursing staff: A study based on the theory of planned behavior. Journal of Management and Organization. 2015;22(1):1-18.

[11] Ekayani N, Wardhani V, Rachmi A. Nurses' intention and behavior in reporting adverse event: Application of theory of planned behavior. Kesmas: National Public Health Journal. 2017;11(3):138144.

[12] Hung C, Lee B, Liang H, Chu T. Factors influencing nurses' attitudes and intentions toward medication administration error reporting. Japan Journal of Nursing Science. 2016;13(3):345-354.

[13] Saranto K, Kinnunen U-M, Koponen S, Kyytsönen M, Hyppönen H, Vehko T. Nurses' competences in informatics/information management as well as experiences/opinions in health and social care information system support for daily practice. FinJeHeW. 2020;12(3): 212-228. 